These articles have been accepted for publication in the British Journal of Dermatology and are currently being edited and typeset. Readers should note that articles published below have been fully refereed, but have not been through the copy-editing and proof correction process. WileyBlackwell and the British Association of Dermatologists cannot be held responsible for errors or consequences arising from the use of information contained in these articles; nor do the views and opinions expressed necessarily reflect those of Wiley-Blackwell or the British Association of Dermatologists

This article is protected by copyright. All rights reserved.

Received Date : 10-Mar-2014

Revised Date : 19-Jun-2014

Accepted Date : 23-Jun-2014

Article type : Cutaneous biology

\title{
Leptin promotes a pro-inflammatory lipid profile and induces inflammatory pathways in human
}

\section{SZ95 sebocytes}

Running head: Leptin in sebaceous gland biology

Manuscript word count: 3.153 (materials and methods not included) table count: - and figure count: 5 with one supplement file online

D. Töröcsik ${ }^{1}$, D. Kovács ${ }^{1}$, E. Camera ${ }^{2}$, M. Lovászi ${ }^{1}$, K. Cseri ${ }^{1}$, G.G. Nagy ${ }^{3}$, R. Molinaro ${ }^{4}$, R. Rühl ${ }^{5}$, G. $\operatorname{Tax}^{6}$, K. Szabó ${ }^{7}$, M. Picardo ${ }^{2}$, L. Kemény ${ }^{6,7}$, C.C. Zouboulis ${ }^{8}$, É. Remenyik ${ }^{1}$

${ }^{1}$ Department of Dermatology, Faculty of Medicine, University of Debrecen, Debrecen, Hungary

${ }^{2}$ Laboratory of Cutaneous Physiopathology and Integrated Centre of Metabolomics Research, San Gallicano Institute of Dermatology, IRCCS, Rome, Italy

${ }^{3}$ Department of Internal Medicine, Faculty of Medicine, University of Debrecen, Debrecen, Hungary ${ }^{4}$ Laboratório de Imunofarmacologia, Instituto Oswaldo Cruz, Fundação Oswaldo Cruz, Rio de Janeiro, Brazil

${ }^{5}$ Department of Biochemistry and Molecular Biology, Faculty of Medicine, University of Debrecen, Debrecen, Hungary 
This article is protected by copyright. All rights reserved.

${ }^{6}$ Department of Dermatology and Allergology, University of Szeged, Szeged, Hungary,

${ }^{7}$ MTA-SZTE Dermatological Research Group, University of Szeged, Szeged, Hungary,

${ }^{8}$ Departments of Dermatology, Venereology, Allergology and Immunology, Dessau Medical Center, Dessau, Germany

Correspondence: Dániel Törőcsik MD PhD,

Address: Department of Dermatology, Faculty of Medicine, University of Debrecen, Debrecen, Hungary, H4032, Nagyerdei krt. 98.

Telephone: +36 52255602

Fax: +36 52255736

Email: dtorocsik@gmail.com

Funding sources: The work was supported in part by TÁMOP 4.2.2.A-11/1/KONV-2012-0031 and National Research Funds OTKA PD101557 projects. D.T. and K. Sz. are recipients of the Bolyai János Fellowship. D.T. has been also awarded the Magyary Zoltán Fellowship (both fellowships by the Hungarian Academy of Sciences), Hungary. E.C. and M.P. were supported by the Italian Ministry of Health through the grant RF-2010-2316435.

The authors have nothing to disclose.

Bulleted statements

What's already known about this topic?

- Leptin is an adipocyte-secreted hormone that regulates weight.

- Leptin is known to link lipid metabolism with inflammation in various cell types. 
This article is protected by copyright. All rights reserved.

- Sebaceous glands are capable of responding to different stimuli by an altered secretion of lipids and production of inflammatory mediators.

What does this study add?

Leptin was recognized as a possible pro-inflammatory player in sebaceous gland biology since sebocytes responded to leptin stimulation with pro-inflammatory changes in the sebaceous lipid profile and with an increased expression of inflammatory enzymes and cytokines.

\section{Summary}

Background: Leptin, the adipocyte-secreted hormone that regulates weight, is known to link lipid metabolism with inflammation in various cell types, however its role in human sebocytes has not yet been investigated.

Objectives: The purpose of the study was to investigate the effects of leptin in human sebaceous gland biology.

Methods: Ob-Rb expression was detected by real time quantitative RT-PCR and by immunochemistry. Lipid analysis was performed by high performance thin layer chromatography, gas-chromatography-mass spectrometry and time of flight mass spectrometer mass detection. Lipid bodies were visualized by BODIPY staining using fluorescent microscopy and measured by flow cytometry. IL-6 and IL-8 mRNA levels were assessed by real time quantitative RT-PCR and their release was evaluated by enzyme-linked immunosorbent assay. COX-2 and 5-LOX protein expressions as well as the phosphorylation of p65 and STAT3 were determined by Western blot analysis.

Results: Expression of $\mathrm{Ob}-\mathrm{Rb}$ was detected in human sebaceous glands and in cultured human SZ95 sebocytes. The treatment of SZ95 sebocytes with leptin led to an enlargement of intracellular lipid bodies, 
This article is protected by copyright. All rights reserved.

increased the ratios of unsaturated/saturated fatty acids and decreased vitamin E levels. Further supporting a pro-inflammatory role, leptin induced COX-2 and 5-LOX expression in SZ95 sebocytes and augmented the production of IL-6 and IL-8 cytokines. Upon leptin treatment, STAT-3 and NF- $\kappa$ B pathways were activated indicating that these known leptin signalling pathways are active in human sebocytes.

Conclusions: Our findings suggest that leptin signalling may be involved in the pro-inflammatory regulation of sebaceous lipid metabolism and in the induction of inflammatory enzymes and cytokines.

\section{Introduction}

Leptin is an adipocyte-derived hormone/cytokine that links nutritional status with neuroendocrine and immune functions ${ }^{1}$. Acting on its receptors in the hypothalamus leptin is a key player in regulating energy intake and expenditure ${ }^{2,3}$. Besides reflecting the amount of energy stored in the adipose tissue, leptin modulates the function of a wide variety of cell types ${ }^{4}$. The full-length form of the leptin receptor $(\mathrm{Ob}-$ $\mathrm{Rb}$ ) is known to be predominantly responsible for active signal transduction leading to modification of intracellular lipid metabolism, activation of several downstream pathways, such as the STAT-3 and the IRS1/PI3K/Akt/NF-kB ones, and an increased production of pro-inflammatory chemokines ${ }^{5-7}$. In addition, leptin increases the expression levels of enzymes involved in the production of inflammatory mediators ${ }^{8,9}$ thus providing an important cross-talk between lipid metabolism and inflammation at the cellular level.

Sebaceous glands and hair follicles form together the pilosebaceous units, whose primary function is the production of sebum ${ }^{10}$. While an altered lipid composition associated with an increased excretion of sebum is one of the most important factors in the development of acne, on the other hand, its impaired production has been proposed as a key feature in atopic dermatitis ${ }^{11}$. Sebaceous glands also play a central role in skin inflammation ${ }^{10,12}$. Pattern recognition receptors such as TLR2, TLR4, and TLR6 are expressed in sebocytes ${ }^{13,14}$, and their activation increases the secretion of both pro- and anti-inflammatory cytokines (IL-6 and IL-10 respectively), chemokines (IL-8), antimicrobial lipids, peptides, periglandular peptides and neuropeptides ${ }^{15-17}$. 
This article is protected by copyright. All rights reserved.

It is conceivable, therefore, that known signalling mechanisms that mediate leptin actions may also be active in sebocytes. Indeed, sebocytes provide an interesting cell model to study abundant, primary lipogenesis and mediation of inflammatory responses ${ }^{18}$.

In this study, we provide evidence that the functionally active full-length form of the leptin receptor $(\mathrm{Ob}-\mathrm{Rb})$ is expressed in human sebaceous glands and in cultured human SZ95 sebocytes and present a systematic analysis on the effect of leptin on SZ95 sebocytes regarding lipogenesis and inflammation.

\section{Materials and methods}

Immunohistochemistry, immunocytochemistry

Anonymised formalin-fixed and paraffin embedded sections of human skin from the tissue archive of the Department of Dermatology, University of Debrecen were acquired after the approval of the Regional and Institutional Ethics Committee, University of Debrecen. SZ95 sebocytes were cultured in chamber slides (LabTek, Thermo Scientific, Rochester, NY), in a density of 10,000 cells/well. Immunohistochemistry and immunocytochemistry was carried out by using a purified primary antibody to the functionally active full length form of the leptin receptor (Ob-Rb; 250739, Abbiotec, San Diego, CA). Staining protocols are presented in supplementary information online.

\section{Cell culture and cell treatment}

The immortalized human sebaceous gland cell line SZ95 was cultured as described previously ${ }^{19}$. Human recombinant leptin (R\&D Systems, Minneapolis, MN) was dissolved and stored at $-70^{\circ} \mathrm{C}$ according to the manufacturer's protocol. For an optimal stimulation, leptin was used at the concentration of $20 \mathrm{nM}$, which is within the normal range of human serum levels ${ }^{6}$. Leptin was monitored for endotoxin contamination using the Limulus Endosafe ${ }^{\circledR}$ KTA2 ${ }^{\mathrm{TM}}$ assay (Charles River Endosafe, Charleston, SC). The endotoxin level was $<0.02 \mathrm{EU}$ per $1 \mu \mathrm{g}$. Parallel experiments were performed using equivalent levels of lipopolysaccharides (LPS; $3 \mathrm{pg} / \mathrm{ml}$ ) that did not result in lipid body enlargement or in STAT3 and p65 phosphorylation (Supplement 1) and did not induce the expression levels of COX-2 or 5-LOX proteins. 
This article is protected by copyright. All rights reserved.

\section{Lipid analyses}

SZ95 sebocytes were cultured in epidermal growth factor-free medium, harvested at the indicated time points, then counted and stored at $-80^{\circ} \mathrm{C}$ until processing. Lipid extraction was performed as previously reported with slight modifications to adapt it to a multiple lipid analyses protocol ${ }^{20}$ (Supplementary information online).

\section{Lipid body detection and enumeration}

Sebocytes were cultured on LabTek chamber slides (Thermo Scientific) and were treated with human recombinant leptin, for 12 and $24 \mathrm{~h}$. Lipid bodies with size over $3 \mu \mathrm{m}$ in SZ95 sebocyte colonies were enumerated with a $\times 100$ objective lens for 100 consecutive cells in each slide (Supplement 2). Staining protocols and information on microscopes and acquisition software are detailed in Supplementary information online.

\section{Western blotting}

Cells were collected at the indicated time points and processed for western bloting according to the protocol shown in the Supplement. Membranes were probed with anti-Ob (MAB867, R\&D Systems), anti-5-LOX (3289; Cell Signaling, Beverly, MA), anti-COX-2 (4842; Cell Signaling), anti-phospho-NFкBp65 Ser536 (3033; Cell Signaling), anti-phospho-STAT-3 Tyr705 (AF4607, R\&D Systems) and anti$\beta$-actin (3700S; Cell Signalling). The Ag-Ab complexes were labelled with appropriate HRP-conjugated secondary Abs (Bio-Rad) and visualized by Immobilon Western HRP Substrate kit (Millipore, Bedford, MA).

\section{ELISA}

Supernatants of SZ95 sebocyte cultures maintained with/without leptin were collected at the indicated time points and were stored at $-20^{\circ} \mathrm{C}$ until they were analyzed for the presence of IL-6, IL-8, TNF $\alpha$ and 
This article is protected by copyright. All rights reserved.

IL-1 $\beta$. Cytokine levels were measured using Quantikine ELISA reagents (R\&D systems) according to the manufacturer's instructions.

Quantitative real-time PCR ( $q R T-P C R)$

To obtain total RNA, cells were centrifuged and pelleted at the indicated time points. RNA isolation, cDNA synthesis, qRT-PCR, used assays and quantification methods are detailed in Supplementary information online.

\section{Statistical analyses}

All data are presented as mean \pm SD. In qRT-PCR experiments, the mean and SD were calculated for both the normalized and the normalizer values. To incorporate the random errors of the measurements, we used the propagation of errors to determine the SD of the normalized values. We made at least four biologic replicates for all experiments. In qRT-PCR experiments, we performed an unpaired, two-tailed, $\mathrm{t}$ test. Differences with $p \leq 0.05$ values were considered statistically significant.

\section{Results}

Ob-Rb is expressed in human sebaceous glands

To address the possible role of leptin signalling in sebaceous gland biology, we first performed immunohistochemical analysis using a specific antibody for the Ob-Rb receptor subtype, which is responsible for active signal transduction in human skin samples of healthy individuals and acne patients. We found that $\mathrm{Ob}-\mathrm{Rb}$ was expressed in the sebaceous glands of both the healthy, as well as the acneinvolved skin (Fig. 1a, b).

Next, we turned our attention to human SZ95 sebocytes and used qRT-PCR, western blotting and immunohistochemical analyses to detect the expression of $\mathrm{Ob}-\mathrm{Rb}$ at mRNA and protein levels. We found that $\mathrm{Ob}-\mathrm{Rb}$ mRNA levels in SZ95 sebocytes were comparable with the levels detected in a human 
This article is protected by copyright. All rights reserved.

immortalized keratinocyte cell line (HaCaT), in primary human keratinocytes (NHEK), as well as in human blood derived monocytes/macrophages (Fig. 1c).

In line with the mRNA analysis, the expression of the $\mathrm{Ob}-\mathrm{Rb}$ protein was detected by western blotting using an antibody that detected also the Ob-Ra variant (Fig. 1d). These results were later confirmed by immunostaining using an antibody specific for the Ob-Rb isoform (Fig. 1e).

These findings confirmed SZ95 sebocytes as a valuable model for further investigations on addressing the role of leptin in sebocyte biology.

\section{Leptin alters lipogenesis in SZ95 sebocytes}

The primary function of sebocytes is their potential to synthetize lipids, therefore we investigated the early and late effects of leptin on the lipid profile of leptin-treated SZ95 sebocytes, assessing the synthesis of major sebaceous lipids such as squalene (SQ), cholesterol $(\mathrm{CH})$, triglycerides $(\mathrm{TG})$, free fatty acids (FFA), as well as alpha-tocopherol (vitamin E, VE). Briefly, cell samples were collected at 12 and $36 \mathrm{~h}$ after leptin treatment and compared to controls using various techniques such as high performance thin layer chromatography (HPTLC), gas-chromatography-mass spectrometry (GC-MS) and high performance liquid chromatography coupled with flight mass spectrometer mass detector (HPLC-ToF/MS).

First, HPTLC with densitometric analysis showed that TG levels were significantly elevated in response to leptin at $36 \mathrm{~h}(+37 \%)$; in contrast to SQ, FFA and $\mathrm{CH}$ levels that did not change significantly (Fig. 2a). For a higher sensitivity and specificity, SQ and $\mathrm{CH}$ levels were simultaneously quantified by GC-MS. The extended analysis showed that while SQ levels were not modified over time in control sebocytes, its concentration tended to increase in the leptin-treated ones. In contrast, concentration of $\mathrm{CH}$ increased in a time-dependent manner in both the control and leptin-treated cells, and leptin treatment did not result in any significant change compared to the controls (Supplement 3).

Next, in order to further investigate representative components of the human sebum such as different FFAs and TG lipid classes, samples were analyzed by HPLC-ToF/MS and GC-MS ${ }^{21}$. The evaluation of the summarized levels and calculated ratios of monounsaturated fatty acids/saturated fatty acids 
This article is protected by copyright. All rights reserved.

(MUFA/SFA) and polyunsaturated fatty acids/saturated fatty acids (PUFA/SFA) showed significant differences in the PUFA/SFA ratios (control vs. leptin at $36 \mathrm{~h}, \mathrm{p}<0.05$ ) and was close to significance regarding the MUFA/SFA ratios (control vs. leptin at $12 \mathrm{~h}, \mathrm{p}=0.07$ ) (Fig. 2b). To assess which FFA ratios changed, an array of 16:1/16:0, 16:2/16:0, 18:1/18:0, 18:2/18:0, 18:3/18:0, 20:1/20:0, 20:2/20:0 and 20:3/20:0 FFA was calculated resulting in significances $(p<0.05)$ and tendencies $(p<0.07)$ (Fig. $2 c)$. The increase in the ratios of selected MUFA/SFA and PUFA/SFA, was confirmed via further analysis of TGs where the calculated ratios of 48:2/48:0 (control $1.69 \pm 0.19$ to $2.06 \pm 0.20)$ and 48:3/48:0 (0.51 \pm 0.07 and $0.66 \pm 0.01)$ changed significantly after $36 \mathrm{~h}$ (data not shown).

The change of the cell redox status consequent to activation of lipid synthesis has barely been investigated in sebocytes. Thus, VE, which accounts for the major lipid scavenger of free radicals in the cell membrane, was also analyzed. The concentration of VE was significantly lower in leptin-treated sebocytes at $36 \mathrm{~h}$ (Fig. 2d).

These data indicate that desaturation of FFA is an early effect of leptin treatment, whereas appreciable induction of TG synthesis occurs at later time points. Moreover a significant decrease in the VE levels indicated that leptin and its lipogenic effects could be associated with consumption of lipophilic antioxidants.

\section{Leptin treatment leads to lipid body enlargement in SZ95 sebocytes}

Sebocytes store and metabolize intracellular lipids in hydrophobic organelles called lipid bodies. In order to detect changes in the lipid body formation we used fluorescent microscopy to visualize the BODIPY stained lipid droplets (Fig. 3a-b). As sebocytes accumulate lipid droplets in high numbers with different sizes, we focused on the lipid droplets exceeding $3 \mu \mathrm{m}$ of size. We found that in the leptin-treated samples the number of cells with such lipid droplets was increased when compared with the untreated controls (Fig. 3c). As BODIPY staining is able to selectively stain lipid droplets it also allows FACS measurements for quantitative analyses ${ }^{22}$. In support to the previous findings, we confirmed that leptintreated SZ95 sebocytes exhibited an increased fluorescence intensity compared to control cells (Fig. 3d). 
This article is protected by copyright. All rights reserved.

Altogether, these results indicate that leptin-treated SZ95 sebocytes display an altered lipid droplet formation.

COX-2 and 5-LOX expression and inflammatory cytokine expression of SZ95 cells is increased by leptin To investigate the potential role of leptin in inflammation, first we measured the protein levels of COX-2 and 5-LOX, the key enzymes in the production of inflammatory mediators in SZ95 sebocytes. Using western blot analysis we could show that the expression levels of COX-2 (Fig. 4a) and 5-LOX (Fig. 4b) were increased upon leptin treatment when compared to untreated cells.

Next, we determined the effect of leptin on the expression of IL-6 and IL-8, shown to be important in the inflammatory signalling of human sebocytes ${ }^{10,15}$. In the presence of leptin the mRNA levels of both IL-6 and IL8 showed a marked up-regulation compared to untreated cells (Fig. 4d). Similar to this, the secreted IL-6 and IL-8 protein levels were also up-regulated, as detected by ELISA measurements (Fig. 4e). On the other hand, we could not detect secretion of TNF $\alpha$ and IL1 $\beta$ by ELISA in any of the tested conditions, a finding that is consistent with previous reports suggesting that SZ95 sebocytes do not secrete these cytokines under normal conditions ${ }^{16,23}$.

To exclude that the measured differences were related to a reduced cell viability we also performed an apoptosis assay at 12, 24 and $48 \mathrm{~h}$ time points and found no differences between the leptin-treated and the untreated control cells (Supplement 4).

These data altogether show that SZ95 sebocytes respond to leptin stimulus with an enhanced expression of inflammatory enzymes and an increased production and secretion of pro-inflammatory cytokines.

Leptin activates STAT-3 and NF- $\mathrm{KB}$ pathways in SZ95 sebocytes

The prime consequence of leptin binding to its receptor is the activation of the STAT-3 pathway leading to STAT-3 phosphorylation ${ }^{24}$, while on the other hand, NF- $\kappa$ B also serves as a downstream signalling 
This article is protected by copyright. All rights reserved.

mediator of leptin ${ }^{25}$. To explore the mechanisms by which leptin could increase the inflammatory response of SZ95 sebocytes we examined its effect on STAT-3 and NF-kB activation. We measured and compared the intensity and length of the phosphorylation of STAT-3 and the NF- $\kappa$ B p65 subunit in the control and leptin-treated SZ95 sebocytes during a 1-hour time period. Upon leptin treatment, both STAT-3 and p65 phosphorylation increased (Fig. 4c), showing that this treatment resulted in an increased STAT-3 and NF-אB activity.

These data suggest that leptin contributes to inflammation through the induction of STAT-3 and NF$\kappa \mathrm{B}$ signalling in SZ95 sebocytes.

\section{Discussion}

Sebaceous glands play a pivotal role in the development of the intact skin barrier through the production of sebum. Moreover, besides their active lipid metabolism ${ }^{26-28}$, sebocytes also play an important role in the setting of an inflammatory environment by being competent for the synthesis and secretion of proinflammatory cytokines, such as IL-6 and IL-8. As a result, they are involved in the initiation of the pathogenesis of sebaceous gland associated diseases such as acne ${ }^{18,29-31}$.

Leptin is an adipokine, secreted predominantly by adipocytes in response to increased lipid uptake ${ }^{32}$. Its serum concentration correlating with the body mass index, and the amount of the adipose tissue is responsible for its systemic effects through the leptin receptor in the hypothalamus. However, the role of leptin is not limited to the regulation of energy expenditure. Besides its metabolic effect, leptin modulates immune responses in many different cell types; adipocytes, macrophages ${ }^{33}$, dendritic cells ${ }^{34}, \mathrm{~T}$ cells $\mathrm{s}^{35}, \mathrm{~B}$ cells $^{36}$, natural killer cells ${ }^{37}$, chondrocytes ${ }^{38}$, fibroblasts ${ }^{39}$ and keratinocytes ${ }^{40}$ all seem to respond to leptin. Regarding its role in skin physiology, leptin was found to be an important player in skin regeneration and ageing, as well as in hair growth ${ }^{39,41,42}$. Considering the complex role of leptin in integrating lipid metabolism and inflammatory responses at a cellular level, it is intriguing to speculate that leptin signalling could be implicated into sebocyte biology, too. Supporting this hypothesis, leptin deficient 
This article is protected by copyright. All rights reserved.

$(\mathrm{ob} / \mathrm{ob})$ mice were found to exhibit an increased sebaceous gland size ${ }^{43}$ with an abnormal production of type II wax diesters, which are important for water repulsion in mice ${ }^{44}$. However, using HE-stained sections for sebaceous gland size measurements and considering the fact that mouse sebum greatly differs from the human one (e.g. contains relatively small amounts of triglycerides ${ }^{45}$ ) conclusions are not easy to be made regarding human sebocyte biology. Moreover, the unavailability of animal models that exert sebaceous gland-associated human diseases, like acne, further suggests that besides the function also the pathology of sebaceous glands have changed with evolution. These findings altogether point towards a possible role for leptin in sebocyte biology, but call for further, cell-type specific studies on how leptin affects lipogenesis and inflammation in the human sebaceous cells.

In our work evidence is provided that leptin receptor is expressed in the human sebaceous glands, besides the human epidermis ${ }^{39}$, human follicular papilla cells ${ }^{46}$ and the sebocyte-like cells of the rat preputial gland ${ }^{47}$, where it was previously detected. Interestingly, the expression of the Ob-Rb receptor was detected both in control and in acne samples, suggesting that these cells can readily respond to leptin under different conditions. In order to investigate how leptin could alter lipogenesis and inflammation in human sebocytes, we confirmed initially the presence of Ob-Rb in SZ95 sebocytes, followed by a comprehensive analysis of the outcomes of leptin stimulus, by measuring the changes in the lipid body formation, lipid profile and the pro-inflammatory responses with the possible signalling pathways behind it (Fig. 5).

Lipid body formation is a phenomenon widely observed in lipid-metabolizing cells, however, their presence and dynamics have only been studied to a limited extent in these cell types ${ }^{20}$. Using BODIPY fluorescent staining, we found that leptin treatment was associated with an enlargement of the lipid droplets and increased fluorescence intensity within SZ95 sebocytes. Interestingly, the same phenomenon could also be observed in macrophages upon leptin treatment ${ }^{48}$, pointing out that leptin could affect intracellular lipid storage and metabolism, and depending on the cell type and model system used, lipolysis is not an obligate outcome alone. Noteworthy, the mechanisms controlling the formation of lipid bodies and the fate of intracellular lipids are likely to be influenced by the specific functions that the 
This article is protected by copyright. All rights reserved.

different tissues carry out, e.g. the adipose tissue accumulates lipids to store excessive energy, whereas lipids being synthetized in the sebaceous glands are excreted to form the skin surface lipid film ${ }^{49}$. Moreover, a possible link between lipid body enlargement and sebum production was already addressed in a recent publication that aimed to characterize sebocytes of Cidea-deficient mice that exerted markedly reduced levels of skin surface lipids. While Cidea-deficient sebocytes with an impaired lipid production and secretion accumulated a large number of smaller-sized lipid droplets, the overexpression of Cidea in human SZ95 sebocytes resulted in an increased lipid storage and in an accumulation of large lipid droplets that were both correlated positively with human sebum secretion. ${ }^{50}$ These results put forward that in the leptin-treated sebocytes the enlargement of lipid droplets could be considered as a marker for increased lipid production and secretion, however more data on the formation, enlargement, function and fate of these lipid bodies are needed to understand their exact role in (leptin-treated) sebocytes.

In order to detect the changes in the levels of lipids that are present in the human sebum, we exploited additional lipidomic analyses ${ }^{21}$ and provided evidence that similarly to other cell types capable of responding to leptin stimulus, leptin altered lipogenesis in SZ95 sebocytes, too. In this process desaturation of FFA might be a key event as revealed by the relative accumulation of different MUFAs and PUFAs. Furthermore, the increased ratios of the unsaturated/saturated FFA could explain the increased levels of TG at the later time points and also the enlargement of lipid bodies as unsaturated FFA were shown to induce TG at greater extents than the saturated ones and promoted TG-enriched lipid body formation in different cell types ${ }^{51}$.

Giving biological relevance to these findings, the importance of the unsaturated/saturated lipid ratio in the sebum was addressed in a former publication showing that a decrease in unsaturated FFA vs. saturated FFA levels in sebum, especially marked by the FFA 16:1/16:0 ratio, was predictive of the clinical improvement in acne, suggesting that the desaturation of sebaceous fatty acids may be involved in acne development ${ }^{52}$. Interestingly, the authors also found that acne subjects on low glycemic load diet (LGL) showed a greater improvement, which correlated with the change in body mass and with the decrease in the levels of unsaturated FFA compared to the increase seen in the control group of patients on non 
This article is protected by copyright. All rights reserved.

LGL $^{52}$. Concluding their findings, they hypothesized that LGL diet may affect sebum composition via undefined metabolic effects in which, according to our understanding, leptin may be a key candidate. In our system, supporting this notion, we provide evidence that leptin might be implicated into the pathophysiology of acne by inducing an "acne-like" change in the sebaceous lipid profile via increasing the amounts of unsaturated FFA, especially the FFA 16:1/16:0 ratio, which largely resembles the changes in the composition of sebum found in acne patients. Moreover, the decrease in the levels of the major sebum antioxidant VE upon leptin treatment, which is also a hallmark in acne patients ${ }^{39}$, further supports a possible role for leptin in acne.

Multiple lines of evidence indicate that the role of sebocytes is not limited to lipogenesis, but is also the modulation of inflammation. Previous reports have shown that, just as it was detected in the sebaceous glands of acne lesions, SZ95 sebocytes also express inflammatory enzymes (COX-2 and 5LOX), cytokines (IL-6) and chemokines (IL-8) at a basal level ${ }^{15}$. Moreover, their expression could be further induced upon treatment with various inflammatory stimuli including arachidonic acid, linoleic acid $^{53}$, and $P$. acnes ${ }^{16}$. We showed that the expression of all these molecules were significantly increased upon leptin treatment supporting that leptin might also be a potential player in inducing inflammatory signalling in human sebocytes.

Tyrosine phosphorylation close to the carboxy-terminus (Y705) and serine phosphorylation at a site within the transactivation domain (S727) of STAT-3 leads to its dimerization, nuclear translocation and DNA binding, resulting in the regulation of its target genes such as c-Myc, JunB, and Mcl-1 ${ }^{54}$. NF- $\kappa \mathrm{B}$ molecules are associated with IкB proteins in the cytoplasm that prevents their nuclear localization. Upon activation, the NF- $\mathrm{kB}$ is released and also translocates to the nucleus with a subsequent DNA binding leading to the expression of genes with specific response elements, in which the phosphorylated p65 subunit of the NF-kB provides the gene regulatory function. By measuring the phosphorylation of STAT3 and p65 after leptin stimulus we found evidence that in leptin-treated SZ95 sebocytes STAT-3 and NF$\kappa \mathrm{B}$ pathways are likely candidates for the mechanisms behind the enhanced inflammatory cytokine production. This is also the first report showing that STAT-3 signalling could be induced in SZ95 
This article is protected by copyright. All rights reserved.

sebocytes, adding important details on the complexity of inflammatory pathways that are active in human sebocytes (Fig. 5).

Based on our findings one might speculate on the association of serum leptin levels with acne. The available data from clinical studies also support that leptin may contribute to sebaceous gland pathology as the serum leptin levels in obese subjects with acne were significantly higher than in obese subjects without acne $e^{55}$. However, no significant differences were observed in non-obese adolescents with or without acne ${ }^{56}$, calling for further studies to define the exact (metabolic) conditions where leptin could affect sebum production and inflammation. Furthermore, since leptin might also be of skin origin, as keratinocytes, fibroblasts and subcutaneous adipocytes were also found to synthetize and secrete leptin upon various stimuli ${ }^{39}$, leptin could also act as a cutaneous transmitter, of which sebocytes are primary targets. Our present work therefore opens new avenues for further investigations that may integrate leptin into the currently modified concepts ${ }^{57}$ of (patho)physiological signalling in sebaceous gland-associated diseases, such as acne.

\section{Conflict of interest}

The authors state no conflict of interest.

\section{Acknowledgements}

This work was supported in part by TÁMOP 4.2.2.A-11/1/KONV-2012-0031 and National Research Funds OTKA PD101557 projects. D.T. and K. Sz. are recipients of the Bolyai János Fellowship. D.T. has been also awarded the Magyary Zoltán Fellowship (both fellowships by the Hungarian Academy of Sciences), Hungary. E.C. and M.P. were supported by the Italian Ministry of Health through the grant RF-2010-2316435. 
This article is protected by copyright. All rights reserved.

\section{References}

1 La Cava A, Matarese G. The weight of leptin in immunity. Nat Rev Immunol 2004; 4:371-9

2 Halaas JL, Gajiwala KS, Maffei M et al. Weight-reducing effects of the plasma-protein encoded by the obese gene. Science 1995; 269:543-6

3 Bjørbaek C, Kahn BB. Leptin signalling in the central nervous system and the periphery. Recent Prog Horm Res 2004; 59:305-31

4 Matarese G, Moschos S, Mantzoros CS. Leptin in immunology. J Immunol 2005; 174:3137-42

5 Santos-Alvarez J, Goberna R, Sánchez-Margalet V. Human leptin stimulates proliferation and activation of human circulating monocytes. Cell Immunol 1999; 194:6-11

6 Mattioli B, Straface E, Quaranta MG et al. Leptin promotes differentiation and survival of human dendritic cells and licenses them for Th1 priming. J Immunol 2005; 174:6820-8

7 Tong KM, Shieh DC, Chen CP et al. Leptin induces IL-8 expression via leptin receptor, IRS-1, PI3K, Akt cascade and promotion of NF-kappaB/p300 binding in human synovial fibroblasts. Cell Signal 2008; 20:1478-88

8 Lam QL, Lu L. Role of leptin in immunity. Cell Mol Immunol 2007; 4:1-13

9 Vuolteenaho K, Koskinen A, Kukkonen M et al. Leptin enhances synthesis of proinflammatory mediators in human osteoarthritic cartilage--mediator role of NO in leptin-induced PGE2, IL-6, and IL-8 production. Mediators Inflamm 2009; 2009:345838 
This article is protected by copyright. All rights reserved.

10 Zouboulis CC, Baron JM, Böhm M et al. Frontiers in sebaceous gland biology and pathology. 2008; Exp Dermatol 17:542-51

11 Zouboulis CC. Acne and sebaceous gland function. Clin Dermatol 2004; 22:360-6

12 Ottaviani M, Camera E, Picardo M. Lipid mediators in acne. Mediators Inflamm 2010; 2010:2010

13 Oeff MK, Seltmann H, Hiroi N et al. Differential regulation of Toll-like receptor and CD14 pathways by retinoids and corticosteroids in human sebocytes. Dermatology 2006; 213:266

14 Lee SE, Kim JM, Jeong MK et al. 11ß-hydroxysteroid dehydrogenase type 1 is expressed in human sebaceous glands and regulates glucocorticoid-induced lipid synthesis and toll-like receptor 2 expression in SZ95 sebocytes. Br J Dermatol 2013; 168:47-55

15 Alestas T, Ganceviciene R, Fimmel S et al. Enzymes involved in the biosynthesis of leukotriene B4 and prostaglandin E2 are active in sebaceous glands. $J$ Mol Med 2006; 84:75-8

16 Nagy I, Pivarcsi A, Kis K et al. Propionibacterium acnes and lipopolysaccharide induce the expression of antimicrobial peptides and proinflammatory cytokines/chemokines in human sebocytes. Microbes Infect 2006; 8:2195-205

17 Linuma K, Sato T, Akimoto N et al. Involvement of Propionibacterium acnes in the augmentation of lipogenesis in hamster sebaceous glands in vivo and in vitro. J Invest Dermatol 2009; 129:2113-9 
This article is protected by copyright. All rights reserved.

18 Zouboulis CC, Jourdan E, Picardo M. Acne is an inflammatory disease and alterations of sebum composition initiate acne lesions. J Eur Acad Dermatol Venereol 2014; 28:527-32

19 Zouboulis CC, Seltmann H, Neitzel H et al. Establishment and characterization of an immortalized human sebaceous gland cell line (SZ95). J Invest Dermatol 1999; 113:1011-20

20 Dahlhoff M, Camera E, Picardo $\mathrm{M}$ et al. PLIN2, the major perilipin regulated during sebocyte differentiation, controls sebaceous lipid accumulation in vitro and sebaceous gland size in vivo. Biochim Biophys Acta 2013; 1830:4642-9

21 Camera E, Ludovici M, Galante M et al. Comprehensive analysis of the major lipid classes in sebum by rapid resolution high-performance liquid chromatography and electrospray mass spectrometry. J Lipid Res 2010; 5111:3377-88

22 Melo RC, D'Ávila H, Bozza PT et al. Imaging lipid bodies within leukocytes with different light microscopy techniques. Methods Mol Biol 2011; 689:149-61

23 Krause K, Schnitger A, Fimmel S et al. Corticotropin-releasing hormone skin signalling is receptormediated and is predominant in the sebaceous glands. Horm Metab Res 2007; 39:166-70

24 Baumann H, Morella KK, White DW et al. The full-length leptin receptor has signalling capabilities of interleukin 6-type cytokine receptors. Proc Natl Acad Sci USA 1996; 93:8374-8

25 Jang PG, Namkoong C, Kang GM et al. NF-kappaB activation in hypothalamic pro-opiomelanocortin neurons is essential in illness- and leptin-induced anorexia. J Biol Chem 2010; 285:9706-15 
This article is protected by copyright. All rights reserved.

26 Makrantonaki E, Zouboulis CC. Testosterone metabolism to 5alpha-dihydrotestosterone and synthesis of sebaceous lipids is regulated by the peroxisome proliferator-activated receptor ligand linoleic acid in human sebocytes. Br J Dermatol 2007; 156:428-32

27 Schuster M, Zouboulis CC, Ochsendorf F et al. Peroxisome proliferator-activated receptor activators protect sebocytes from apoptosis: a new treatment modality for acne? Br J Dermatol 2011; 164:182-6

28 Chen W, Tsai SJ, Wang CA et al. Human sebocytes express prostaglandin E2 receptors EP2 and EP4 but treatment with prostaglandin E2 does not affect testosterone production. $B r$ J Dermatol 2009; 161:674-7

29 Ganceviciene R, Graziene V, Fimmel S et al. Involvement of the corticotropin-releasing hormone system in the pathogenesis of acne vulgaris. Br J Dermatol 2009; 160:345-52

30 Kamp S, Fiehn AM, Stenderup K et al. Hidradenitis suppurativa: a disease of the absent sebaceous gland? Sebaceous gland number and volume are significantly reduced in uninvolved hair follicles from patients with hidradenitis suppurativa. Br J Dermatol 2011; 164:1017-22

31 Logan AC. Linoleic and linolenic acids and acne vulgaris. Br J Dermatol 2008; 158:201-2

32 Fain JN, Leffler CW, Cowan GS Jr et al. Stimulation of leptin release by arachidonic acid and prostaglandin E(2) in adipose tissue from obese humans. Metabolism 2001; 50:921-8

33 Batra A, Okur B, Glauben R et al. Leptin: a critical regulator of CD4+ T-cell polarization in vitro and in vivo. Endocrinology 2010; 151:56-62 
This article is protected by copyright. All rights reserved.

34 Mattioli B, Straface E, Matarrese P et al. Leptin as an immunological adjuvant: enhanced migratory and CD8+ T cell stimulatory capacity of human dendritic cells exposed to leptin. FASEB $J$ 2008; 22:2012-22

35 Kim SY, Lim JH, Choi SW et al. Preferential effects of leptin on CD4 T cells in central and peripheral immune system are critically linked to the expression of leptin receptor. Biochem Biophys Res Commun 2010; 394:562-8

36 Agrawal S, Gollapudi S, Su H et al. Leptin activates human B cells to secrete TNF- $\alpha$, IL-6, and IL-10 via JAK2/STAT-3 and p38MAPK/ERK1/2 signalling pathway. J Clin Immunol 2011; 3:472-8

37 Tian Z, Sun R, Wei H et al. Impaired natural killer (NK) cell activity in leptin receptor deficient mice: leptin as a critical regulator in NK cell development and activation. Biochem Biophys Res Commun 2002; 298:297-302

38 Figenschau Y, Knutsen G, Shahazeydi S et al. Human articular chondrocytes express functional leptin receptors. Biochem Biophys Res Commun 2001; 287:190-7

39 Poeggeler B, Schulz C, Pappolla MA et al. Leptin and the skin: a new frontier. Exp Dermatol 2009; 19:12-8

40 Kanda N, Watanabe S. Leptin enhances human beta-defensin-2 production in human keratinocytes. Endocrinology 2008; 149:5189-98

41 Frank S, Stallmeyer B, Kämpfer $\mathrm{H}$ et al. Leptin enhances wound re-epithelialization and constitutes a direct function of leptin in skin repair. J Clin Invest 2000; 106:501-9 
This article is protected by copyright. All rights reserved.

42 Sumikawa Y, Nakajima T, Inui S. Leptin is a paracrine regulator of hair cycle. J Invest Dermatol 2008; 128:S146-S876.

43 Chen HC, Smith SJ, Tow B et al. Leptin modulates the effects of acyl CoA:diacylglycerol acyltransferase deficiency on murine fur and sebaceous glands. J Clin Invest 2002; 109:175-81

44 Thody AJ, Shuster S. Control and function of sebaceous glands. Physiol Rev 1989; 69:383-416.

45 Nikkari T. Comparative chemistry of sebum. J Invest Dermatol 1974; 62:257-67

46 Iguchi M, Aiba S, Yoshino $\mathrm{Y}$ et al. Human follicular papilla cells carry out nonadipose tissue production of leptin. J Invest Dermatol 2001; 117:1349-56

47 Deplewski D, Qin K, Ciletti N et al. Unique mode of lipogenic activation in rat preputial sebocytes. $J$ Nutr Metab 2011; 2011:163631

48 Maya-Monteiro CM, Almeida PE, D'Avila $\mathrm{H}$ et al. Leptin induces macrophage lipid body formation by a phosphatidylinositol 3-kinase- and mammalian target of rapamycin-dependent mechanism. $J$ Biol Chem 2008; 283:2203-10

49 Camera E, Mastrofrancesco A, Ludovici M et al. Comprehensive analysis of intact sebaceous lipids to investigate the fate of free fatty acids in human sebocytes. J Invest Dermatol 2010; 130:S14

50 Zhang S, Shui G, Wang G et al. Cidea control of lipid storage and secretion in mouse and human sebaceous glands. Mol Cell Biol 2014; 34:1827-38 
This article is protected by copyright. All rights reserved.

51 Mei S, Ni HM, Manley S et al. Differential roles of unsaturated and saturated fatty acids on autophagy and apoptosis in hepatocytes. J Pharmacol Exp Ther 2011; 339:487-98

52 Smith RN, Braue A, Varigos GA et al. The effect of a low glycemic load diet on acne vulgaris and the fatty acid composition of skin surface triglycerides. J Dermatol Sci 2008; 50:41-52

53 Zouboulis CC, Angres S, Seltmann H. Regulation of stearoyl-coenzyme A desaturase and fatty acid delta-6 desaturase-2 expression by linoleic acid and arachidonic acid in human sebocytes leads to enhancement of proinflammatory activity but does not affect lipogenesis. Br J Dermatol 2011; 165:26976

54 Ding BB, Yu JJ, Yu RY et al. Constitutively activated STAT-3 promotes cell proliferation and survival in the activated B-cell subtype of diffuse large B-cell lymphomas. Blood 2008; 111:1515-23

55 Abulnaja KO. Changes in the hormone and lipid profile of obese adolescent Saudi females with acne vulgaris. Braz J Med Biol Res 2009; 42:501-5

56 Kaymak Y, Adisen E, Ilter $\mathrm{N}$ et al. Dietary glycemic index and glucose, insulin, insulin-like growth factor-I, insulin-like growth factor binding protein 3, and leptin levels in patients with acne. J Am Acad Dermatol 2007; 57:819-23

57 Zouboulis CC, Eady A, Philpott M et al. What is the pathogenesis of acne? Exp Dermatol 2005; 14:143-52 
This article is protected by copyright. All rights reserved.

\section{Figures legends}

Figure 1. Leptin receptor $\mathrm{Ob}-\mathrm{Rb}$ is present in the sebaceous glands of normal and acne-involved skin and is expressed in SZ95 sebocytes. Leptin receptor Ob-Rb protein immunostaning of human sebaceous glands in control (a) and acne (b) skin samples as described in Materials and Methods. Original magnification $\times 200$. (c) Leptin receptor $\mathrm{Ob}-\mathrm{Rb}$ mRNA expression in HaCaT keratinocytes, NHEK keratinocytes, human blood derived monocytes/macrophages, and SZ95 sebocytes determined by RT-Q-PCR. All PCR data are expressed as a ratio of the leptin receptor $\mathrm{Ob}-\mathrm{Rb}$ transcripts relative to $\beta$ actin expression. The expression levels are representative of three independent measurements. Error bars indicate the SD of the relative expression. (d) Western blot analysis of leptin receptors $\mathrm{Ob}-\mathrm{Rb}$ and $\mathrm{Ob}-\mathrm{Ra}$ by using an antibody that targets both $\mathrm{Ob}-\mathrm{R}$ isoforms. The images are representative of three different blots. (e) Digitized images of immunofluorescently labeled SZ95 sebocytes stained with selective Ob-Rb antibody. Scale bar: $10 \mu \mathrm{m}$.

Figure 2. Leptin affects lipogenesis in SZ95 sebocytes by increasing triglyceride levels, increasing the ratios of unsaturated/saturated fatty acid levels and by decreasing the level of vitamin $E$ (a) Densitometric analysis of bands (squalene (SQ), triglycerides (TG), free fatty acids (FFA), cholesterol $(\mathrm{CH})$ obtained by HPTLC (average \pm SD of normalized intensity) of lipid extracts from SZ95 sebocytes treated with leptin for the indicated time points, showing that leptin induced the levels of triglycerides at a significant extent at $36 \mathrm{~h}\left({ }^{*} \mathrm{p}<0.05\right)$. (b) FFA were quantified following separation with HPLC and detection with a ToF/MS detector at the respective treatment time with leptin (12 and $36 \mathrm{~h})$. Ratios of MUFA/SFA and PUFA/SFA were calculated showing a significant change in the case of PUFA/SFA (CTR vs. LEPT at 36h, p <0.05) and being close to significance in the case of MUFA/SFA (c) Array of calculated ratios of representative human sebum components ranging from 16:1/16:0, 16:2/16:0, 18:1/18:0, 18:2/18:0, 18:3/18:0, 20:1/20:0, 20:2/20:0 and 20:3/20:0 resulting in significances $(\mathrm{p}<0.05)$ and tendencies $(\mathrm{p}<0.07)$ showing that leptin affected the distribution of FFA. [ ${ }^{*} \mathrm{p} \leq 0.05 * * \mathrm{p} \leq 0.005$ (leptin vs. control)]. (d) Concentration of vitamin E (alpha-tocopherol) in SZ95 sebocytes was significantly 
This article is protected by copyright. All rights reserved.

lower in leptin treated sebocyte cells at $36 \mathrm{~h}(* \mathrm{p} \leq 0.05)$ as determined by GC/MS. The data were reported mean $\pm \mathrm{SD}$ of the concentrations determined in 4 independent experiments.

Figure 3. Leptin treatment increased lipid body size. Representative images of BODIPY and a blue nuclear counterstained SZ95 sebocytes that were (a) untreated or (b) treated with leptin and observed by fluorescence microscopy as described in Materials and Methods (original magnification, X100). Red arrows point to lipid bodies with an increased size that are characteristic for leptin-treated cells. Enumeration of lipid bodies with a size exceeding $3 \mu \mathrm{m}$ (c) in untreated and leptin-treated SZ95 sebocytes at 12 and $24 \mathrm{~h}$ after stained with BODIPY and a blue nuclear counterstain. 4X100 cells from groups were analysed in each sample as detailed in Supplement 2. Bars represent the mean \pm SEM from four independent experiments. Intensity of BODIPY staining was determined by flow cytometry on SZ95 sebocytes (d) treated with vehicle (Control) (filled gray) or leptin (black line). Numbers are the mean fluorescence intensity values. One representative experiment of four performed is shown.

Figure 4. Leptin treatment increases COX-2 and 5-LOX expression, activates STAT-3 and NF-кB signalling and increases the secretion of inflammatory cytokines. Western blot analysis of (a) COX-2 (b) 5-LOX and phosphorylation of (c) STAT-3 Tyr705 and NF-кBp65 Ser536 as well as $\beta$-actin expression in untreated (control) and leptin-treated SZ95 sebocytes for the indicated periods of time. The images are representative of at least three different blots. (d) The mRNA levels for IL-6 and IL8 in SZ95 sebocytes stimulated with leptin for $2 \mathrm{~h}$. Expression levels were determined by real-time quantitative RTQ-PCR. Data are expressed as a ratio of the transcripts relative to $\beta$-actin expression. Error bars indicate the $\mathrm{SD}$ of the relative expression. ${ }^{*} \mathrm{p}<0.03$, compared with the respective control value. (e) Relative protein levels of IL-6 and IL-8 from supernatants of SZ95 sebocytes treated with leptin for the indicated time points were determined by ELISA as described in Materials and Methods. Data were normalized with protein levels similarly determined from untreated SZ95 sebocytes of the same experiment (treated/untreated). Data show the means and SEM of five independent experiments. Significance versus untreated cells $* * \mathrm{p}<0.05$ 
This article is protected by copyright. All rights reserved.

Figure 5. Leptin integrates into sebocyte biology - mechanisms involved in the leptin-treated SZ95 sebocytes. Activation with leptin is resulted in the up-regulation of inflammatory enzymes COX-2 and 5LOX and in the increased expression and secretion of inflammatory cytokines IL- 6 and IL- 8 that is likely to be underpinned by the activation of the STAT -3 and NF- $\kappa \mathrm{B}$ pathways. Treatment with leptin also resulted in an altered lipogenesis by increasing the ratio of monounsaturated fatty acids/saturated fatty acids (MUFA/SFA) and polyunsaturated FFA/saturated FFA (PUFA/SFA) and reducing the level of vitamin E. The enlargement of lipid droplets upon leptin treatment correlated with the increased levels of unsaturated FFA and TG.

\section{Figure 1.}
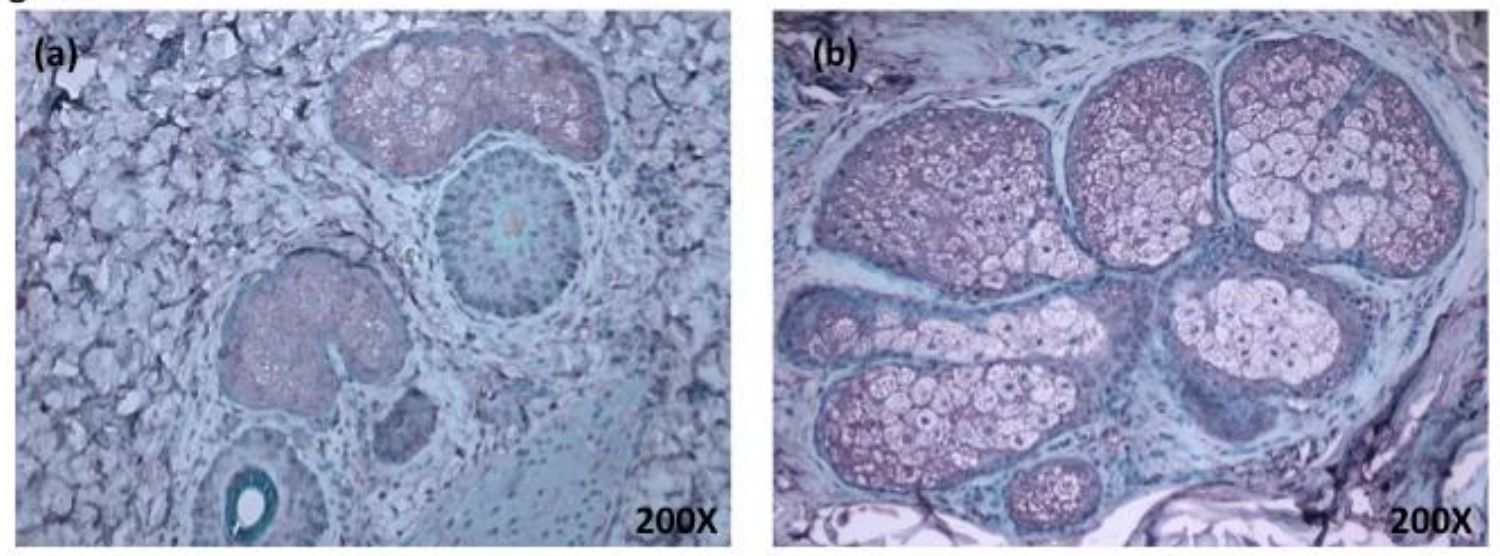

(c)

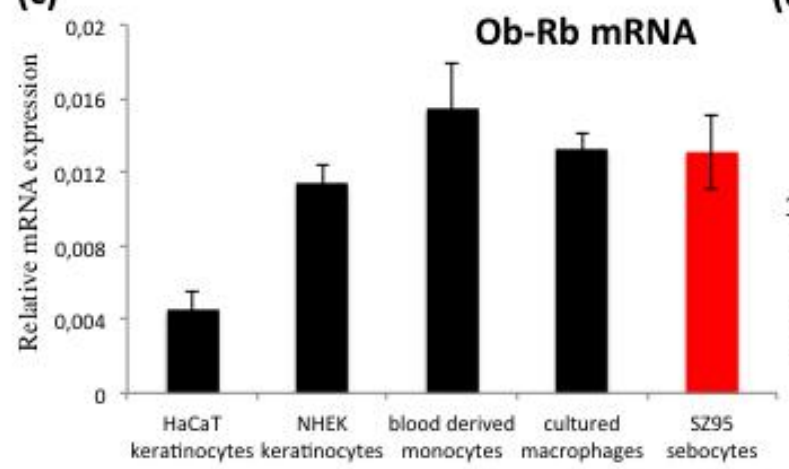

(d)

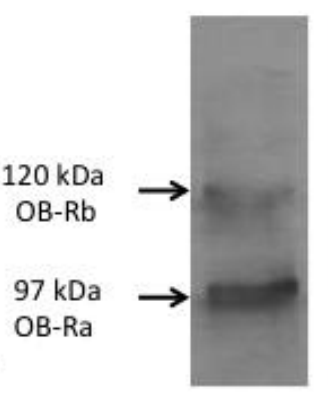

(e)

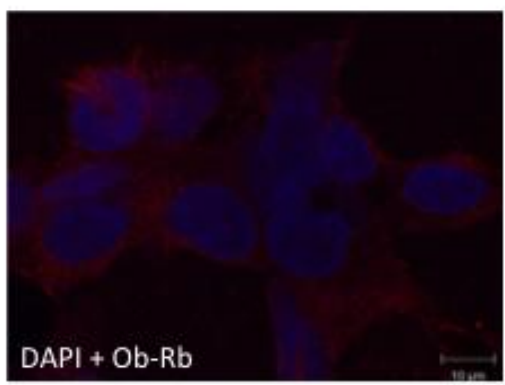


Figure 2.

(a) Densitometry

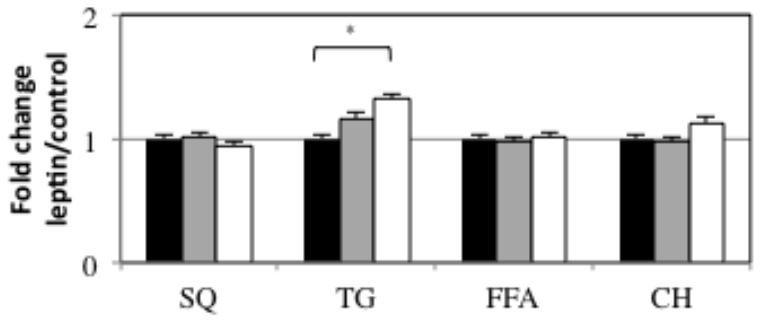

(b)

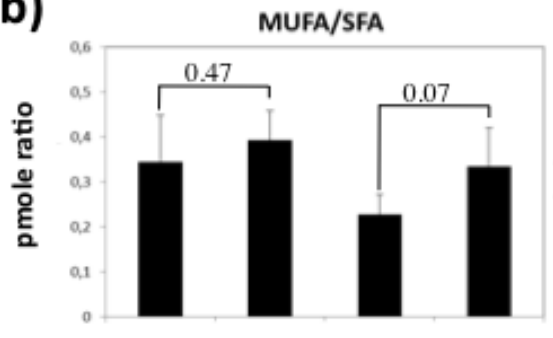

(c)
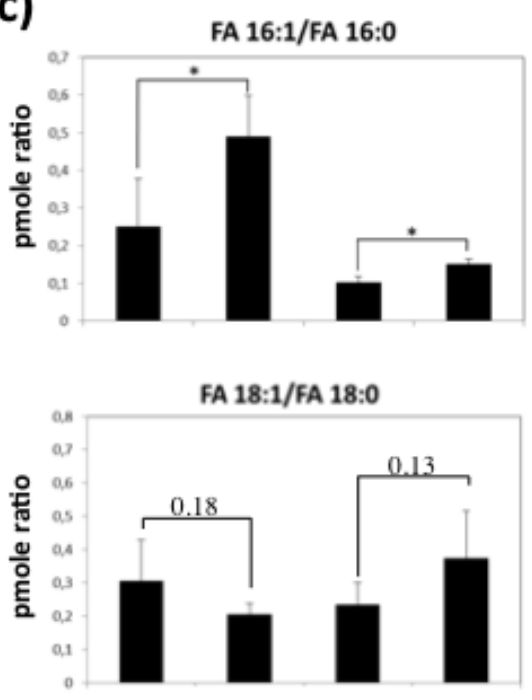

FA 20:1/FA 20:0

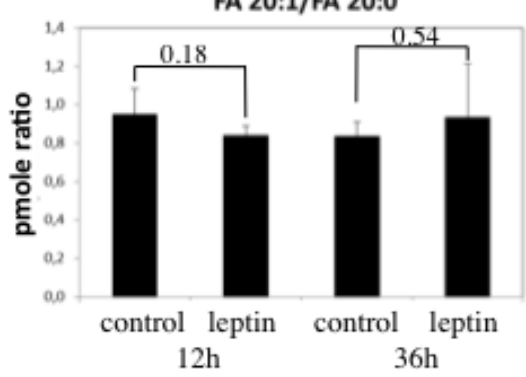

(d)

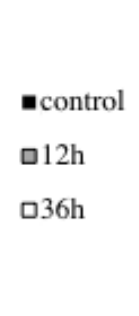

PUFA/SFA

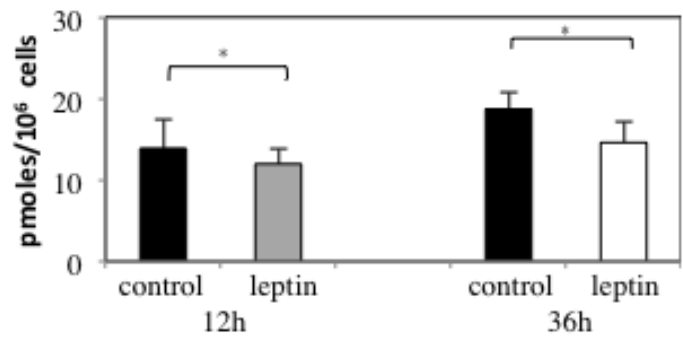

Vitamin E

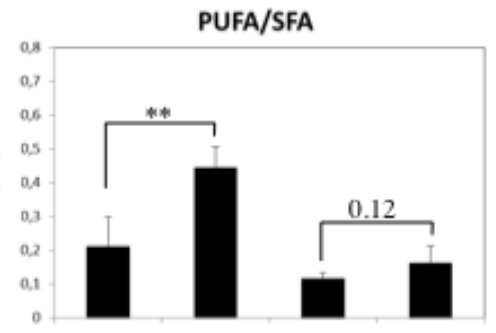

FA 16:2/FA 16:0

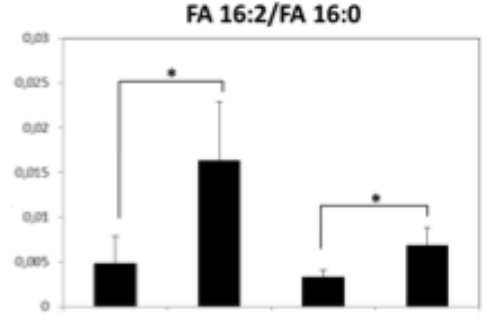

FA 18:2/FA 18:0

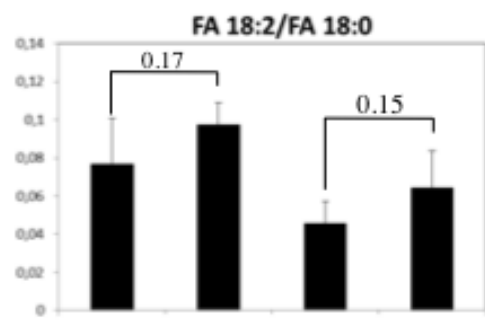

FA 20:2/FA 20:0

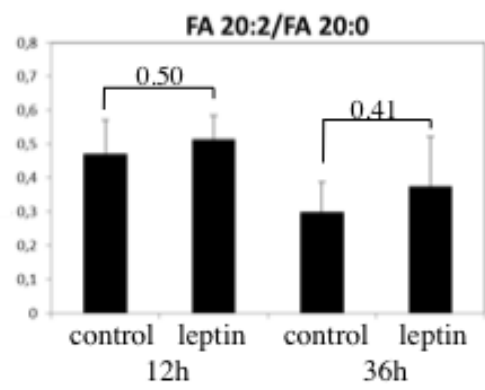

$* p<0.05$

${ }^{* *} p<0.005$
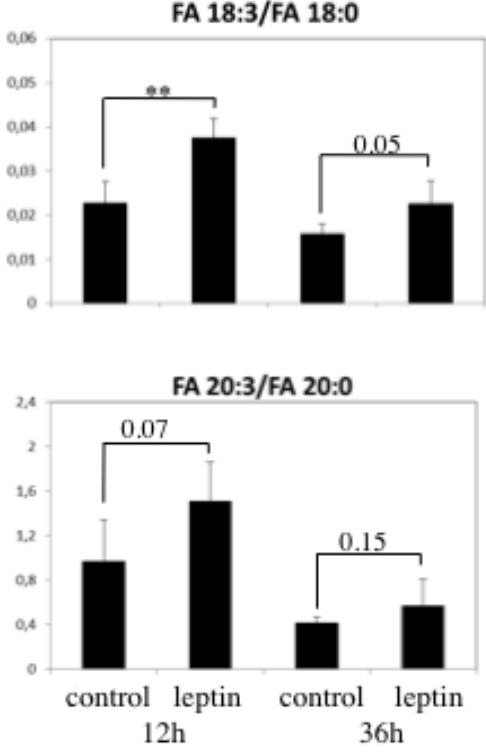
This article is protected by copyright. All rights reserved.

Figure 3.
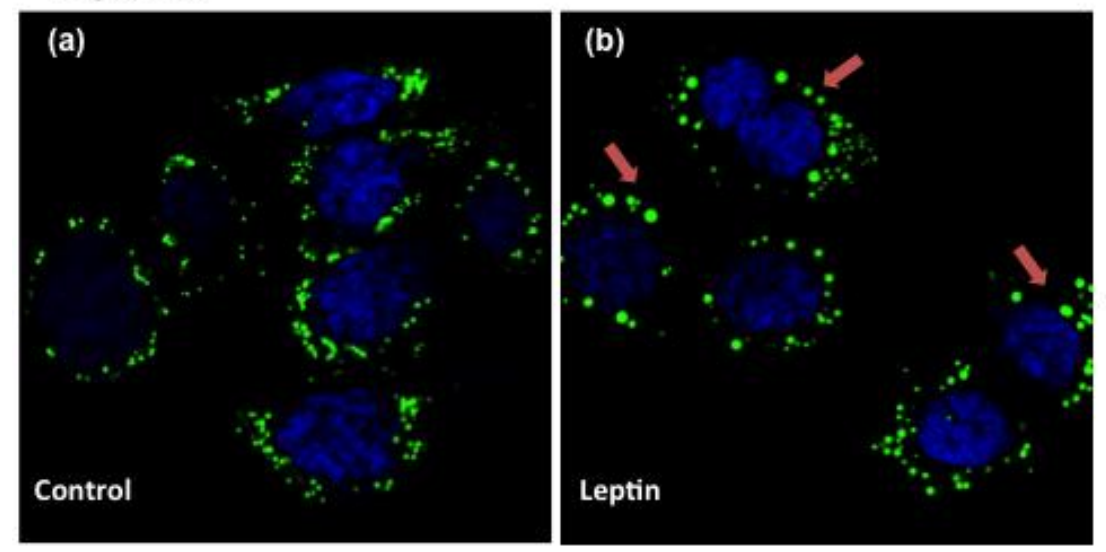

(d)

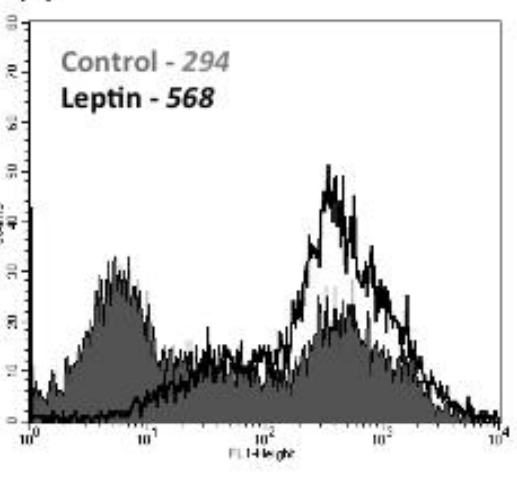

(c)
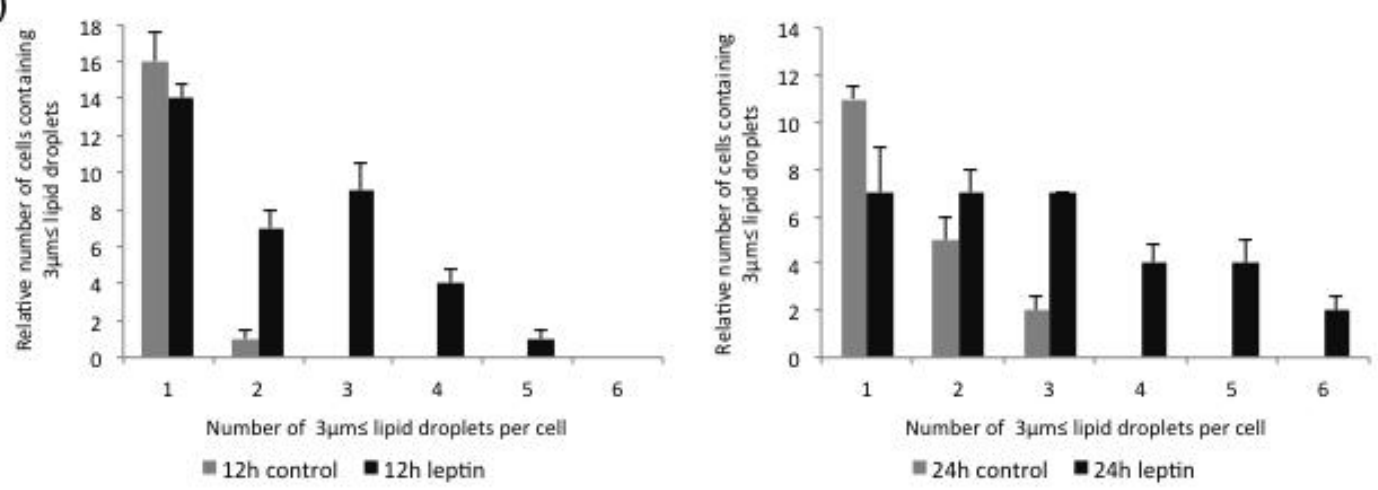
This article is protected by copyright. All rights reserved.

Figure 4.

(a)

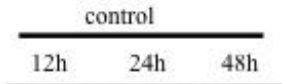

COX-2 $\beta$-actin

(b)
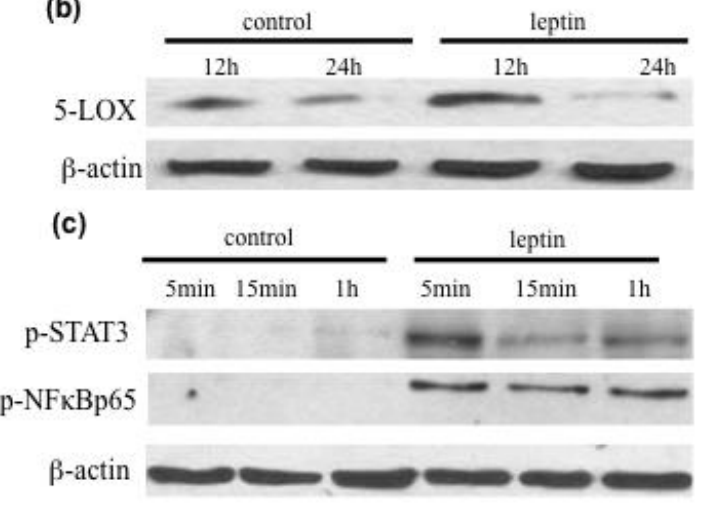

$\beta$-actin
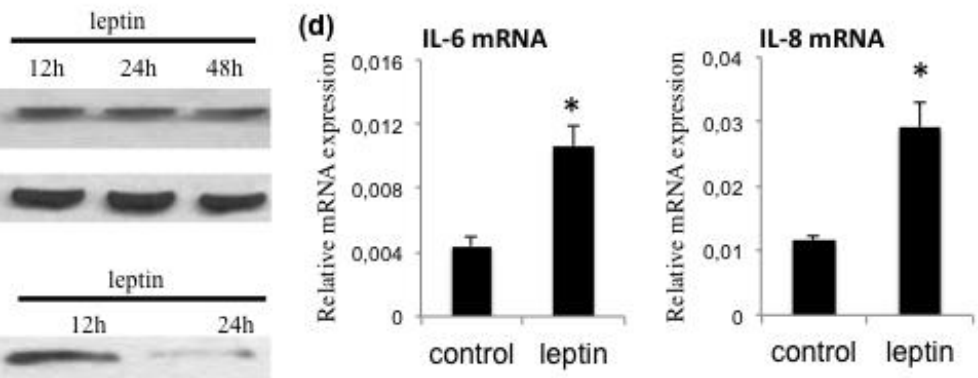

(e)
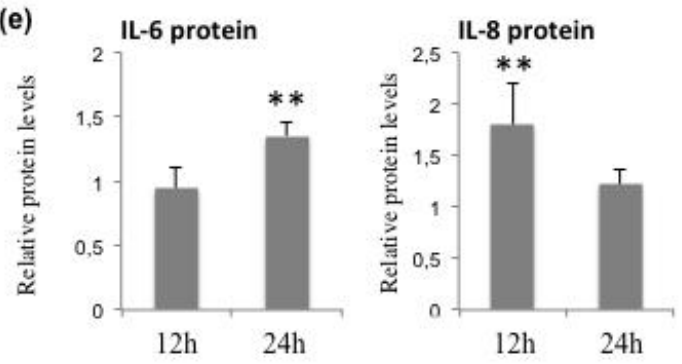

Figure 5.

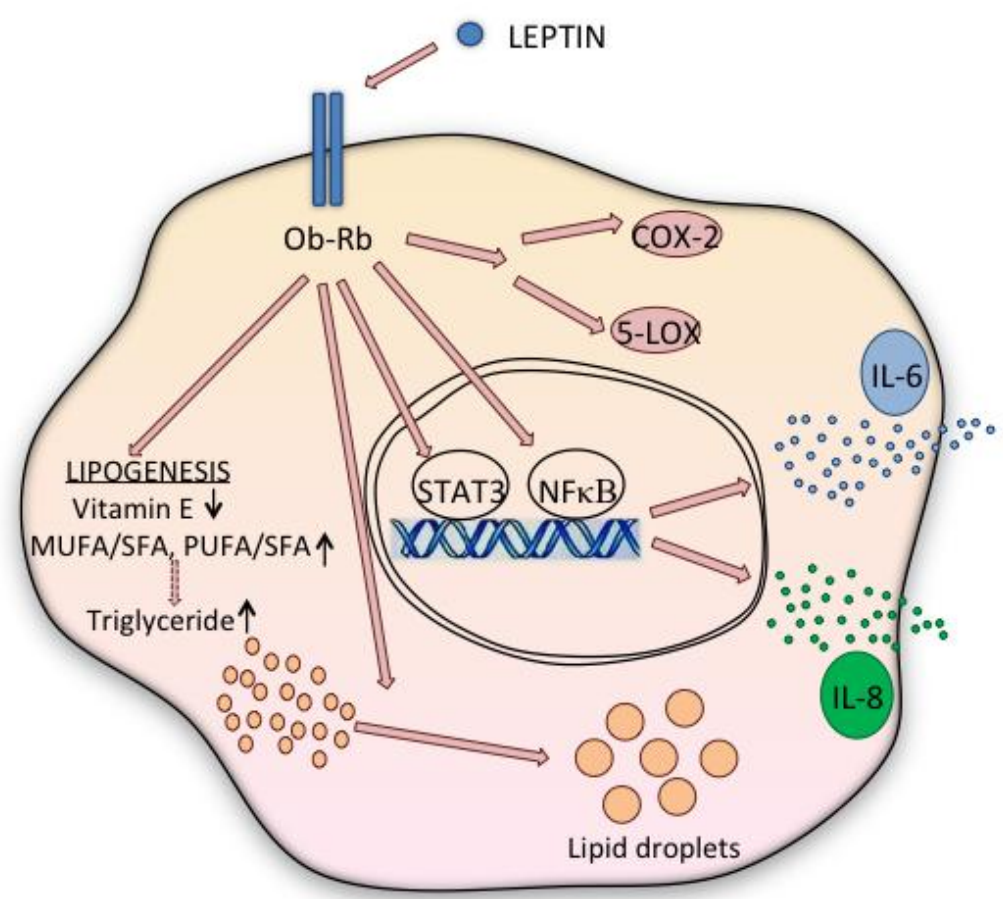

